\title{
Venus, an Astrobiology Target
}

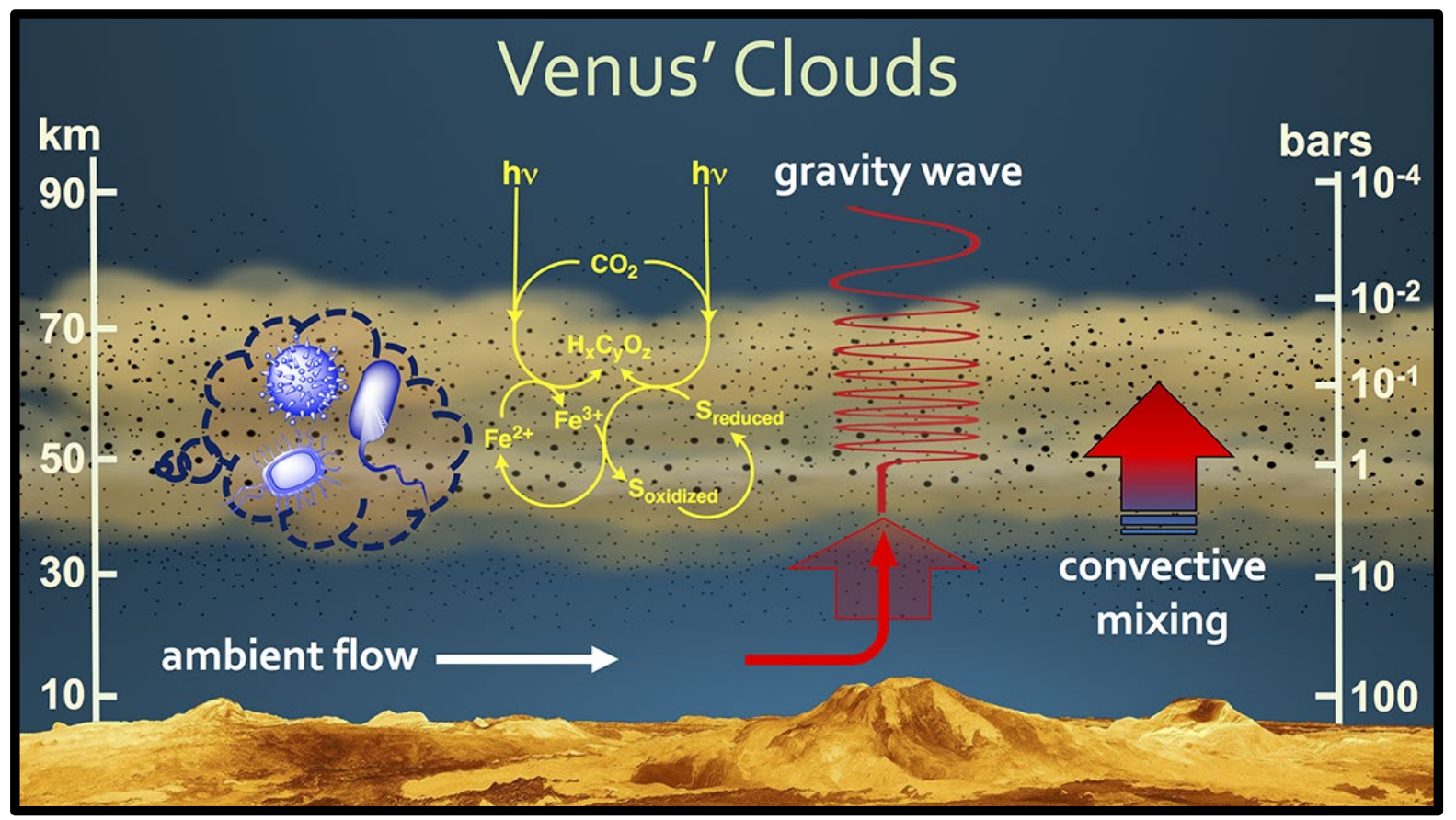

A schematic representation summarizing processes in Venus' atmosphere that may support a cloud-based biosphere in Venus' lower clouds. Adapted from - Limaye et al. (Astrobiology, doi/10.1089/ast.2017.1783).

Sanjay S. Limaye, University of Wisconsin, 1225 West Dayton Street, Madison, WI 53706, Telephone: (608) 262-9541, E-mail: sslimaye@wisc.edu

Mark A. Bullock, Science and Technology Corp. Kevin H. Baines, CalTech/JPL and UW-Madison Charles Cockell, University of Edinburgh Jaime A. Cordova, University of Wisconsin James A. Cutts, CalTech/JPL Diana Gentry, NASA/ARC

David Grinspoon, Planetary Science Institute James Head, Brown University Kandis Lea Jessup, SwRI-Boulder Vladimir Kompanichenko, Insitute for Complex Analysis, RAS, Birobidzhan, Russia

Yeon J. Lee, Technical Uni. of Berlin, Germany
Richard Mathies, University of California, Berkeley

Tetyana Milojevic, Vienna University, Austria Rakesh Mogul, Cal Poly Pomona

Rosalyn A. Pertzborn, University of Wisconsin Lynn Rothschild, NASA/ARC

Satoshi Sasaki, Tokyo University of Technology

Dirk Schulze-Makuch, Technical University of Berlin/IFG, Germany

Sara Seager, Massachusetts Institute of Technology

David J. Smith, NASAIARC Michael J. Way, NASA/GISS

Endorsed by 
Executive Summary: Beyond Earth, the closest planetary environment that could possibly support life are the clouds of Venus. With Earth-like pressures and temperatures, the acidic cloud aerosols could potentially host extremophiles such as acidophilic archaea that are among the oldest organisms on Earth found in considerably less acidic environment. We present the case that Venus meets the first strategic objective, "Understand the Sun, Earth, Solar System, and Universe" (Discovering the Secrets of the Universe and Searching for Life Elsewhere), in NASA's strategic plan for explorationa and should be considered as an astrobiology target. Venus is an accessible analog to explore the diversity and evolution of the habitability of exoplanets, which is drawing a lot of interest at present. In this paper we make a case for undertaking biosignature measurements along with other chemical and dynamical analyses in the Venus clouds. This white paper is based on the presentations and discussions completed during the Cloud layer Habitability of Venus Workshop held in October $2019^{\mathrm{b}}$ and represents a broad consensus view of the nearly fifty scientists from diverse disciplines who attended the workshop.

Introduction. NASA's strategic plan mandates that "research is conducted that will enable the search for signs of life on worlds that may be capable of harboring life, both within our own Solar System (SS) and within the galaxy". The plan goes on to state the importance of research that improves techniques and develops ideas for discovering and characterizing habitable and/or inhabited environments on planets in and beyond the SS, coupled with an understanding of what could lead to potential false positives for habitability or life. Research into the essential elements needed to identify the timeline on which any SS body may have maintained conditions needed for life to emerge, remain or be suspended will enable prioritization of exploration within the solar system and of exoplanets for targeted follow-up observations.

The search for life in the solar system and beyond is guided by our ability to understand how life originated and flourished on Earth, our single known example. Though many hypotheses for 'weird' life with different biochemical foundations have been put forward [1], Earth's history nevertheless remains our best roadmap for the potential for life. At present, this means that the projected timeline of current and past habitability on any Solar System object is dependent primarily on its water history, informed by how closely the emergence and sustainability of its theoretical habitats is consistent with our current understanding of habitats and biological processes on Earth. This understanding develops in parallel with our ever-expanding understanding of Earth's history, evolution, and habitability.

Beyond Earth, the closest planetary environment proposed to support life is the clouds of Venus. At present, the timeline of Venus' past and current surface, subsurface, and cloud habitability is ill-defined. To advance our understanding of these issues, investigations are needed that focus on (i) understanding the anticipated timeline of life emergence as it relates to Venus' geological water history record, relative to Earth's water history and life emergence record; (ii) consistency of any region of Venus' surface or atmosphere with environmental conditions required for the emergence and sustainability of microbial life as we currently understand it, based on Earth habitats that may be partially analogous; and (iii) characterization of the potentially detectable properties of microbes observed on Earth that are known to thrive under similar conditions - including constant and intermittent stresses and water, energy, and trace molecule availability found in Venus' atmosphere (or surface, or subsurface).

Water history and planetary evolution. The conditions at the surface of Venus today are hostile to organic molecules and to most bio-signatures as they are currently understood (other than isotopes). The $\mathrm{D} / \mathrm{H}$ ratio in Venus' atmosphere below the clouds is about 150 times that of Earth's [2, 3], suggesting that Venus lost a great deal of water to space. This was the first hint that Venus may have had a watery past. $\mathrm{D} / \mathrm{H}$ ratio above the clouds has been found to be $240 \pm 25$ times higher than Earth's [4], implying even greater loss of water to space. Today we see water escaping from Venus via $\mathrm{H}+$ and $\mathrm{O}+$ ions [5]. Kasting [6] modeled the

\footnotetext{
${ }^{\mathrm{a}} \mathrm{https} / / /$ science.nasa.gov/science-red/s3fs-public/atoms/files/nasa_2018_strategic_plan_0.pdf

${ }^{\mathrm{b}} \mathrm{http}: / /$ venera-d.cosmos.ru/index.php?id=workshop2019\&L $=2$
} 
early atmosphere of Venus and concluded that it would have taken at least 600 My for Venus to lose an ocean, if it indeed had one. Extending Kasting's 1988 work, Bullock and Grinspoon [7] and Way et al. [8] concluded that clouds and atmospheric dynamics of slowly rotating worlds (like Venus) would mitigate atmospheric temperatures and water loss, and an ocean could have lasted several Gy; these conclusions were recently reaffirmed [9].

In the 1990s the Magellan spacecraft mapped the entire surface of Venus with radar, revealing its geologic record and inspiring investigations into the evolutionary history of the planet. The small number and relatively even distribution of impact craters superposed on the surface seen in Magellan images is interpreted to mean that the average surface age is between 300 and 700 My old (McKinnon et al., 1997). Global geologic mapping [10] showed that something has erased the first $\sim 80-90 \%$ of the geologic record, and that the subsequent geologic history is characterized by decreasing intensity of tectonism [11] and volcanism [12]. Modeling the early atmosphere of Venus with a 1D Radiative Transfer model [13] and cloud formation showed that the last drops of a warm ocean may not have evaporated. A strong possibility exists that Venus had oceans for a geologically long period of time - perhaps more than the 600 My into midNoachian Mars. If life arose on Venus similarly to Earth, believed to be near hydrothermal vents [14], it would have been forced to adapt to the gradual desiccation of the planet and the redistribution of its volatiles. The tessera terrains are believed to be some of the oldest rocks on Venus although the absolute age is unknown and different units may have formed at different times. Their rock composition holds the clues for the past presence of water and thus habitability and evolution of life.

We need better knowledge about the surface of Venus regarding rock ages (especially in tessera terrains), history of water loss and surface mineralogy. This would allow us to answer the question: Did early Venus have the conditions necessary for life to arise, based on comparable assumptions made about other astrobiological targets such as Mars, Europa, and Enceladus?

Habitability and the limits of life. Venus is unique among rocky planets in the solar system for its dense, persistent cloud cover, which maintains Earth-like pressures and temperatures at some altitude ranges. Thus, although present-day Venus surface conditions are hostile to organic molecules and to most (nonisotopic) bio-signatures as understood - notwithstanding speculation about life in super-critical fluids or subsurface water refugia [15] - the possibility of persistently airborne microbial species at Venus has been postulated multiple times over the decades [16-20]. Interestingly, the "inversion" model which proposes the origin of life through responses to incessant physic-chemical oscillations in the surroundings may also provide support to the possibility of life [21, 22].

The potential for abiogenesis during Venus' early wet period is supported by the potential similarities to Earth in the same time frame. Life may also have been delivered to Venus through transfer of Earth (or Martian) biogenic matter [23]; interplanetary matter transfer has occurred consistently throughout the solar system history up to the present day [24, 25]. In each case, either or both of which may have occurred [26], the past and present-day habitability of the atmosphere can be evaluated in light of life in extreme environments on Earth.

Lessons from Earth indicate that 'life finds a way' when faced with challenging extremes (e.g., low temperature, or extreme $\mathrm{pH}$ ) [27-29]. For Venus, survival in the aqueous and acidic atmospheric aerosols would represent such a challenge. If life became established on an early, wet Venus, as water was lost, it would have had to adapt to increasingly desiccating conditions as the oceans evaporated with the resulting acidic aerosols in the atmosphere serving as the remaining bodies of habitable liquid water. As such, life may have adapted to survival in the warm aerosol layer in Venus' clouds [18]. Alternatively, life may have been seeded by meteoritic or cometary dust, where habitation of Venus' clouds was dependent upon adaptation to multiple extremes [30]. Similarly, if indeed the surface conditions on Venus became hostile due to extreme warming and loss of surface water, life would have experienced multiple extreme conditions in the 
atmosphere. Thus, the duration of the period (if any) when both the putative ocean(s) and the permanent cloud layer were present is a key astrobiology question.

Venus' global, layered cloud cover consists mainly of three different sized particles [31] with mean diameters ranging from $\sim 0.5 \mu \mathrm{m}$ (Mode 1), $\sim 2-3 \mu \mathrm{m}$ (Mode 2) and $>3 \mu \mathrm{m}$ (Mode 3 ). The exact composition of the smallest particles, which are likely spherical, is uncertain but suggested to be sulfuric acid solutions containing various minerals. These are more prevalent at higher altitudes, with the 2-3 $\mu$ m diameter particles found throughout the cloud layer, and the largest particles found near the bottom of the cloud layer [32]. The abundance of water peaks be near the base of the clouds - at $42 \mathrm{~km}$, and is about $0.52 \%$ mole fraction [33]. The temperature range across the lower clouds is well within growth limits for terrestrial microbes [34], from $\sim 100{ }^{\circ} \mathrm{C}$ at $47 \mathrm{~km}$ to $\sim-20{ }^{\circ} \mathrm{C}\left(-18\right.$ to $\left.-22{ }^{\circ} \mathrm{C}\right)$ at $62 \mathrm{~km}$; similarly, pressure [35, 36], UV and radiation flux [16, 37], and photonic energy [38] do not appear to be limiting, and a hypothetical redox-based nutrient cycling has been proposed $[18,20]$. Although the effective $\mathrm{pH}$ of the aerosols can be interpreted differently given the extremes of $>80 \%$ sulfuric acid [17, 39], even under the most generous range of -1.5 to 1 , it is near the limit where only a few lineages of archaea [40] and eukaryotic acidophiles such as Cyanidium [29] are known to survive. The low water activity of $<0.02$ [41] is substantially below that of even the most saturated brines [42] and at best on par with Earth's driest deserts [43]. Although the measured size of Venus aerosols, particularly in the larger lower-level regimes, is compatible with the $0.2-1 \mu \mathrm{m}$ typical size of terrestrial microbes [44], measurements of aerosol shape, size and residence time would be a key factor for active research [39], especially in light of the typically long generation times (weeks to months) for many terrestrial extremophiles.

By comparison, the aerial component of Earth's biosphere, and its impact on atmospheric, ecological, and biochemical cycling, has been recognized for some time [45-47]. As far as is currently known, airborne microbes on Earth are transitory and always return to the surface via wet or dry deposition. Though metabolic activity has been shown in short-term cloud droplets [48] and indirectly indicated in some laboratory work [49], in situ airborne reproduction has not yet been detected. Earth's stratospheric sulfate layer, a population of supercooled sulfuric acid and water aerosols (acid weight fraction $0.6-0.85$, size $0.1-1 \mu \mathrm{m}$ [50], density 0.1 to $1 \mathrm{~cm}^{-3}$ [51]) is perhaps the closest match to an Earth analogue environment in terms of chemistry, lack of water, and anthropogenic isolation. This environment has so far yielded only a handful of bioaerosol samples limited to dormant cells or spores hardened against long-term desiccation and UV exposure. Some of these adaptions include protective pigments that absorb strongly in the NUV [52]. This is discussed further below on spectral signatures.

In total, therefore, the open questions regarding Venus' habitability may be summarized as follows:

1. Are there sufficient basic nutrients $(\mathrm{C}, \mathrm{H}, \mathrm{N}, \mathrm{O}, \mathrm{P}, \mathrm{S})$ in the cloud aerosols? $\mathrm{C}, \mathrm{H}, \mathrm{N}, \mathrm{O}$ and $\mathrm{S}$ are abundant, but phosphorus has been detected only once, with some uncertainty over interpretation [53].

2. Is there sufficient water activity in the cloud aerosols for either continuous or sporadic growth and reproduction/division? Potential analogues include the dry conditions of Earth's Atacama desert or stratospheric sulfate aerosol layer.

3. Were habitats continuously available from the putative origin of life in surface oceans to the present low water content cloud cover?

4. Is the UVC and cosmic radiation flux in the potentially habitable altitude range limiting?

5. Are there organics or biosignatures present in the atmosphere? A significant and puzzling amount of $\mathrm{CH}_{4}$ was detected by the Pioneer Venus Large Probe [54]. Recently, $\mathrm{PH}_{3}$ has been suggested as a potential biosignature for exoplanets [55] - is it present in the cloud layer of Venus?

6. Is the residence time of Venus aerosols long enough for putative microbes to reproduce/divide? A very recent study explores this question in some detail [39] for Venus. 
7. Can microbes reproduce/divide while airborne?

8. What are the survival strategies, protective mechanisms and metabolisms of spore-forming and non-spore-forming microorganisms while airborne, including pigmentation and high UV absorption?

9. Are there sufficiently available energy sources such as light or chemical redox couples to allow for life?

10. What are the shapes of the larger cloud particles as can be determined from microscopic imaging?

These questions drive the need for more lab and analog studies of extremophile species. They also drive the need for Venus in-situ measurements of habitability requirements such as basic life nutrients, particularly phosphorus, as well as bio-precursor species and even biosignatures. Several techniques may be selected/developed to implement this including high-resolution $X$-ray fluorescence. Constraining the residence time potential will also require continued and more detailed understanding of vertical transport and turbulent processes in Venus' atmosphere. Advancement in these modeling efforts requires that future Venus exploration prioritizes acquisition of winds and temperature data retrievals at altitudes extending at least through the cloud layers ( 45 to $75 \mathrm{~km}$ ) on a global basis (i.e., all latitudes, longitudes and local times). This may be achieved via a combined observing effort utilizing remote and in-situ observation techniques. Funding for basic research, technology development, lab studies, analog field-work and mission opportunities supporting these investigations will allow us to answer in the next decade the question: What is the potential for modern-day Venus to harbor microbes or signatures of past life?

Spectral Considerations. Another key element of this conversation is that the potential habitability of Venus' clouds may be linked to a long-standing mystery on Venus-what is the chemical composition and nature of the absorber that produces strong absorption at $365 \mathrm{~nm}$ supporting strong spectral contrasts between the absorbing material and Venus' high albedo $\mathrm{H}_{2} \mathrm{SO}_{4}$ clouds [56]? A large number of candidates have been proposed (Table 1), but none have been able to explain the absorption or observed properties of the contrasts. Some terrestrial microorganisms compatible with Venus' chemical cloud composition (such as iron-rich acidophiles) have spectral properties that appear to mimic the observed spectral response of Venus cloud tops between 300 and $400 \mathrm{~nm}$, leading to the proposal that putative Venus microorganisms are possible contributors to the low ultraviolet albedo of Venus[18]. Given that the identity and nature of the absorbers responsible for the bulk of the solar energy absorbed by Venus in its atmosphere and clouds remain unknown, resolution of its identity is a high priority and a link to the potential of life must also be considered.

Figure 1 shows the spectrum of Venus [57] between

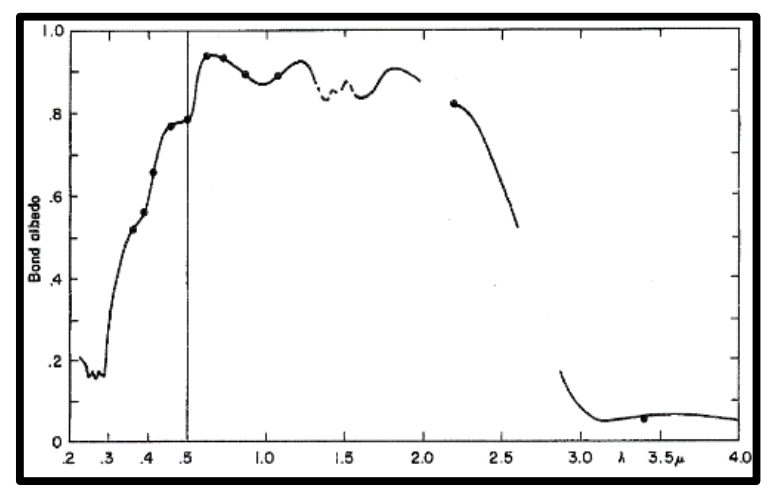

Figure 1: Disk averaged spectrum of Venus showing the strong absorption below $500 \mathrm{~nm}$. The disk resolved spectra of the planet show noticeable differences over latitudes and phase angles.

280 and $4000 \mathrm{~nm}$. The albedo spectrum indicates increasing absorption below about $500 \mathrm{~nm}$. Weaker absorption occurs over a wider range of wavelengths beyond $500 \mathrm{~nm} . \mathrm{SO}_{2}$ and $\mathrm{SO}$ gas absorption is responsible for the distinct absorption bands observable below $330 \mathrm{~nm}$ [58]; and an underlying continuum absorption from an unknown source is also anticipated below $300 \mathrm{~nm}[59,60]$. Although Figure 1 shows that only weak absorptions occur longward of $400 \mathrm{~nm}$-it is important to know if chemical species producing the underlying continuum absorption shortward of $300 \mathrm{~nm}$ also contribute weakly to absorption properties evident longward of $400 \mathrm{~nm}$ or even combine with the $\mathrm{CO}_{2}$ and $\mathrm{H}_{2} \mathrm{O}$ absorptions identified at visible and NIR wavelengths. The decrease beyond $2.5 \mu \mathrm{m}$ is not fully understood [61]. This can only be resolved by 
identifying the composition and nature of the unknown continuum absorption source at the short wavelengths and potential absorbers at long wavelengths. It is likely there is more than one chemical or biological species, as has been postulated by multiple researchers over the decades [62-64]. Spatial/spectral contrasts patterns may be used as a key constraint on absorbing species properties. For example, the evolution of the absorbers responsible for the observed contrasts over short [65] and long timescales [66, 67] is a critical consideration in the quest for the source of the solar absorption in the Venus clouds. The key question may be why the contrasts exist at all if the physical properties of the absorbers are not very different from the $\sim 1 \mu \mathrm{m}$ radius particles-what creates the difference? And are these differences evidence of non-uniformity in particle mixing, chemical differentiation, or differentiation between biogenic and non-biogenic sources?

Table 1: Solar radiation absorbers proposed or detected in the Venus atmosphere.

\begin{tabular}{|c|c|c|c|c|}
\hline Number & Absorber & Reference & Absorption Range (nm) & Remarks \\
\hline 1 & $\mathrm{SO} 2$ & Barker (1975) & $240-330$ & \\
\hline 2 & so & & UV & \\
\hline 3 & $\cos$ & Krasnopolsky, 1986 & 240 & \\
\hline 4 & $\mathrm{CS}_{2}$ & Barker (1978), Young (1978) & & \\
\hline 4 & $\begin{array}{l}\text { Disulfur Oxide and disulfur } \\
\text { dioxide (OSSO) }\end{array}$ & $\begin{array}{l}\text { Frandsen et al. 2017, Perez- } \\
\text { Hoyos et al., 2018, Wu et al. } \\
2018\end{array}$ & $320-400$ & $\begin{array}{l}\text { Wu et al. (2018). OSSO abunbdances do not match } \\
\text { photochemical model (Kranspolsky 2019) }\end{array}$ \\
\hline 5 & $\mathrm{~S}_{2} \mathrm{O}$ & $\begin{array}{l}\text { Hapke and Graham (1985, } \\
\text { 1989) }\end{array}$ & $310-470,650-740$ & \\
\hline 6 & $\mathrm{~S}_{8}$ & $\begin{array}{c}\text { Hapke and Nelson (1975), } \\
\text { Young (1977) }\end{array}$ & $320-500$ & $10 \mu \mathrm{m}$ size grains of morphous sulfur \\
\hline 7 & Amorphous sulfur $(\mathrm{S} 3, \mathrm{~S} 4)$ & Toon et al., 1982 & UV & \\
\hline 8 & $\mathrm{SCl}_{2}$ & Krasnopolsky, 1986 & $270-450$ & \\
\hline 9 & $\begin{array}{l}\text { Ammonium pyrosulfite. } \\
(\mathrm{NH} 4)_{2} \mathrm{~S}_{2} \mathrm{O}_{5}\end{array}$ & Titov 1983 & $320-500$ & \\
\hline 10 & Nitrosylsulfuric acid $\left(\mathrm{NOHSO}_{4}\right)$ & Watson et al. 1979 & $250-500$ & \\
\hline 11 & Carbon suboxide polymer & Sinton (1953), Shimizu, 1977 & 215 & Possible indicators of life? (Shimizu, 1977) \\
\hline 12 & $\mathrm{Cl}_{2}$ & Pollack et al. 1980 & $190-500$ & \\
\hline 13 & Croconic acid $-\mathrm{C}_{5} \mathrm{H}_{5} \mathrm{O}_{2}$ & Hartley et al. 1989 & $220-420$ & \\
\hline 14 & NO & Shaya and Caldwell 1975 & $215,226.5$ & $222.5 \mathrm{~nm}$ band never observed \\
\hline 15 & $\mathrm{~N}_{2} \mathrm{O}$ & Shaya and Caldwell 1975 & $170-220$ & $\begin{array}{l}\text { Abundance should reach } 10^{-4} \text { of } \mathrm{CO}_{2} \text { to explain } \\
\text { absorption at } 200 \mathrm{~nm} \text { (Shimizu, 1977) }\end{array}$ \\
\hline 16 & $\mathrm{H}_{2} \mathrm{~S}$ & Shaya and Caldwell 1975 & $180-230$ & \\
\hline 17 & $\mathrm{HNO}_{3}$ & Shaya and Caldwell 1975 & 2000,300 & $\begin{array}{l}\text { If mixed with } \mathrm{H}_{2} \mathrm{SO}_{4} \text {, absorption shifts to } 260 \mathrm{~nm} \\
\text { (Shimizu, 1977) }\end{array}$ \\
\hline 18 & $\mathrm{FeCl}_{3}$ & Zasova et al. 1987 & $300-450$ & \\
\hline 19 & $\mathrm{FeCl}_{2}$ & Kuiper, 1969 & UV & \\
\hline 20 & Perchloric acid $\left(\mathrm{HClO}_{4}\right)$ & Zahn et al. (1983) & UV & \\
\hline 21 & Anhydrous silicate & Pollack et al. (1975) & $2700-4000$ & \\
\hline 22 & Fe-S based microorganisms & Hapke and Nelson (1975) & & Could be significant for radiation budget \\
\hline \multirow[t]{2}{*}{23} & Photosynthetic microorganisms & Grinspoon (1997) & & \\
\hline & & Schulze-Makuch et al. (2004) & & \\
\hline 24 & $\begin{array}{c}\text { Hypothetical Chemolithotrophic } \\
\text { Microorganisms }\end{array}$ & Limaye et al. (2018) & Broad spectrum & $\begin{array}{l}\text { Many terrestrial biotic materials and proteins have } \\
\text { absorption features comparable to Venus spectrum }\end{array}$ \\
\hline
\end{tabular}

When considering the potential associations of the optical attributes of the cloud layer with candidate microbial species, the properties of the candidate microbe species must be characterized sufficiently that we are be able to ascertain if these species exhibit either singly. or in concert with other non-biogenic sources, the cloud patterns observed at Venus. Advancement in these efforts will require a combined observing effort utilizing remote and in-situ observation techniques that trace Venus' spectral response and aerosol characteristics on a global basis (i.e., all latitudes, longitudes and local times) over a broad range of altitudes, including at a minimum the cloud layer altitudes ( 45 to $75 \mathrm{~km})$. Given the impact of the absorber on Venus' overall energy budget and climate, funding and mission opportunities supporting this work must be prioritized in the next decade!

Summary. In summary, there are three key reasons Venus is of great interest as an astrobiology target. First, the strong likelihood that liquid water was present on Venus' rocky surface for periods that are 
empirically long enough for the emergence of life. Second, the potential for survival of life until the present day in the extreme environment of the clouds, partially supported by factors that have been well constrained $(T, P, U V)$ with other factors under investigation $\left(\mathrm{pH}, \mathrm{a}_{w}\right.$, residence time). Third, the unexplained observations of properties, particularly absorptive properties in the clouds, similar to those of some terrestrial biogenic materials. These three points make a case for exploring Venus as a past and potentially current habitable world. To complete this will require undertaking biosignature measurements along with other chemical and dynamical analyses in the clouds of Venus. As our nearest neighbor, Venus is also an important resource for exploring the diversity, evolution, and potential habitability of terrestrial exoplanets [68], including its transition from early potential habitability to a seemingly uninhabitable surface. These investigations are aligned with VEXAG investigations I-A.HO and AL; II-B.AE and UA) and I III-B.Cl, and are key drivers for understanding Venus, and should be prioritized in the next decade of Venus exploration.

A longer version of this paper is under review for the Astrobiology journal's Special Collection on Venus.

Acknowledgements. This contribution was made possible by research supported by NASA Grant NNX16AC79G (Limaye), NASA Grant NNX16AK82G (Jessup), and NASA Grant NNX16AC81G (Bullock).

\section{References}

1. Space Studies Board, N.R.C., The limits of organic life in planetary systems. 2007: National Academies Press.

2. Donahue, T.M., Icarus, 1999. 141: p. 226-235.

3. Donahue, T.M., et al., Science, 1982. 216: p. 630-633.

4. Fedorova, A., et al., Journal of Geophysical Research (Planets), 2008. 113( (E5) ff): p., pp.E00B22.

5. Persson, M., et al., Geophysical Research Letters, 2018. 45: p. 10,805.

6. Kasting, J.F., Icarus, 1988. 74(3): p. 472-494.

7. Bullock, M.A., et al., Icarus, 2001. 150: p. 19-37.

8. Way, M.J., et al., Geophysical Research Letters, 2016. 43: p. 8376-8383.

9. Way, M.J., et al., Journal of Geophysical Research: Planets, 2020. 125(5): p. e2019JE006276.

10. Ivanov, M.A., et al., Planetary and Space Science, 2011. 59(13): p. 1559-1600.

11. Ivanov, M.A., et al., Planetary and Space Science, 2015. 113-114: p. 10-32.

12. Ivanov, M.A., et al., Planetary and Space Science, 2013. 84: p. 66-92.

13. Grinspoon, D.H., et al., Did Venus Experience One Great Transition or Two?, in AAS/Division for Planetary Sciences Meeting Abstracts \#35. 2003. p. 44.03.

14. Russell, M.J., et al., Terra Nova, 1993. 5(4): p. 343-347.

15. Schulze-Makuch, D., et al., Astrobiology, 2002. 2: p. 197-202.

16. Cockell, C.S., Planetary and Space Science, 1999. 47: p. 1487-1501.

17. Grinspoon, D., et al., in Exploring Venus as a Terrestrial Planet. 2007. p. 191-206.

18. Limaye, S.S., et al., Astrobiology, 2018. 18(9): p. 1181-1198.

19. Morowitz, H., et al., Nature, 1967. 215: p. 1259.

20. Schulze-Makuch, D., et al., Astrobiology, 2004. 4: p. 11-18.

21. Kompanichenko, V.N., Thermodynamic Inversion. Thermodynamic Inversion, ISBN 978-3-319-53510-4. Springer International Publishing AG, 2017, ed. K. Langeheinecke. 2017: Springer, Cham.

22. Kompanichenko, V.N., Geosciences, 2019. 92(9(2)): p. 11.

23. Melosh, H.J., et al., Meteoritics, 1993. 28: p. 398.

24. Carrillo-Sánchez, J.D., et al., Icarus, 2020. 335: p. 113395.

25. Plane, J.M.C., et al., Space Science Reviews, 2018. 214: p. 23.

26. Izenberg, N.R., et al. arXiv e-prints, 2020. arXiv:2007.00105.

27. Cavalazzi, B., et al., Astrobiology, 2019. 19(4): p. 553-578.

28. Mesbah, N.M., et al., Applied and Environmental Microbiology, 2012. 78(12): p. 4074-4082. 
29. Rothschild, L.J., et al., Nature, 2001. 409(6823): p. 1092-1101.

30. Seckbach, J., et al. 2015. p. 153-170.

31. Ragent, B., et al., Advances in Space Research, 1985. 5(11): p. 85-115.

32. Titov, D.V., et al., Space Science Reviews, 2018. 214(8): p. 126.

33. Oyama, V.I., et al., Journal of Geophysical Research, 1980. 85: p. 7891-7902.

34. Domagal-Goldman, S.D., et al., Astrobiology, 2016. 16(8): p. 561.

35. Kato, C., et al., Applied and environmental microbiology, 1998. 64(4): p. 1510-1513.

36. Nicholson, W.L., et al., Applied and environmental microbiology, 2010. 76(22): p. 7559-7565.

37. Dartnell, L.R., et al., Icarus, 2015. 257: p. 396-405.

38. Raven, J., et al., Astrobiology, 2006. 6(4): p. 668-675.

39. Seager, S., et al., Astrobiology, 2020. Accepted.

40. Johnson, D.B., et al., Advances in microbial physiology, 2008. 54: p. 201-255.

41. Gentry, D., et al., Venus aerosol sampling considerations for in site biological analysis, in Venera-D Landing Sites and Cloud Layer Habitability Workshop. 2019: Space Research Institute, Moscow.

42. Bolhuis, H., et al., BMC genomics, 2006. 7(1): p. 169.

43. Kieft, T.L., in Encyclopedia of Environmental Microbiology, G. Bitton, Editor. 2003, John Wiley \& Sons, Inc.: Hoboken, NJ, USA. p. 1-25.

44. Knollenberg, R., et al., Journal of Geophysical Research: Space Physics, 1980. 85(A13): p. 8039-8058.

45. Amato, P., et al., PLoS One, 2017. 12(8): p. e0182869.

46. Delort, A.-M., et al., Atmospheric Research, 2010. 98(2-4): p. 249-260.

47. Pratt, K.A., et al., Nature Geoscience, 2009. 2(6): p. 398-401.

48. Amato, P., et al., Scientific Reports, 2019. 9(1): p. 4383.

49. Sattler, B., et al., Geophysical Research Letters, 2001. 28(2): p. 239-242.

50. Yue, G., et al., Journal of Geophysical Research: Atmospheres, 1994. 99(D2): p. 3727-3738.

51. Ovigneur, B., et al., Atmospheric Measurement Techniques, 2011. 4(11): p. 2359.

52. Yang, W., et al., Frontiers in Plant Science, 2020.11.

53. Andreichikov, B., et al., Kosmicheskie Issledovaniia, 1987.

54. Donahue, T.M., et al., Geophysical research letters, 1993. 20(7): p. 591-594.

55. Sousa-Silva, C., et al., Astrobiology, 2020. 20(2): p. 235-268.

56. Esposito, L.W., et al., in Venus, D.M. Hunten, Editor. 1983. p. 484-564.

57. Kuiper, G., et al. in Bulletin of the American Astronomical Society. 1969.

58. Barker, E.S., Geophysical Research Letters, 1979. 6: p. 117.

59. Jessup, K.-L., et al., Icarus, 2020. 335: p. 113372.

60. Krasnopolsky, V.A., Icarus, 2012. 218(1): p. 230-246.

61. Krasnopolsky, V.A., in Venus, D.M. Hunten, Colin, L., Donahue, T.M., Moroz, V.I., Editor. 1983, The University of Arizona Press: Tucson, Arizona. p. 459-483.

62. Pérez-Hoyos, S., et al., Journal of Geophysical Research (Planets), 2018. 123: p. 145.

63. Pollack, J.B., et al., Journal of Geophysical Research: Space Physics, 1980. 85(A13): p. 8141-8150.

64. Travis, L.D., Journal of Atmospheric Sciences, 1975. 32: p. 1190-1200.

65. Limaye, S.S., et al., Planetary and Space Science, 2019: p. 104710.

66. Lee, Y.J., et al., Icarus, 2015. 253: p. 1-15.

67. Lee, Y.J., et al., The Astronomical Journal, 2019. 158(3): p. 126.

68. Kane, S.R., et al., Journal of Geophysical Research: Planets, 2019. 124(8): p. 2015-2028. 